\title{
Radiation-Induced Phenomena in Wide-Gap Laser Materials Used in High-Radiation Areas
}

\author{
V.V. Harutyunyan*, E.M. Aleksanyan, V.V. Arzumanyan, A.O. Badsakyan \\ A.I. Alikhanyan National Science Laboratory (Yerevan Physics Institute), \\ 0036, Yerevan, Alikhanian Br. Street, Armenia \\ Email: vharut@yerphi.am
}

(Received: September 27, 2021; Revised: October 10, 2021; Accepted: October 15, 2021)

\begin{abstract}
The analysis of the surface states in experimental reflection spectra is carried out peculiarities of the formation of defects of the electronic structure of irradiation corundum crystals is considered. It is shown that change of crystals reflectional ability is related to radiative growth forced by aluminum ions. The processes of radiative growth depend on the type and energy of irradiating high-energy particles. Kinetic of that growth is presented, which relates the intensity of zones with free aluminum ions to the concentration of such zones in near-surface layer.
\end{abstract}

Keywords: reflection spectra of corundum single crystals, electrons, reactor neutrons, MeV/amu lead ions, radiation damage, point, complex $\left[\mathrm{Al}_{\mathrm{i}} \mathrm{F}\right]$, clustered defects, lattice defects

https://doi.org/10.52853/18291171-2021.14.3-142

\section{Introduction}

The study of radiation effects in solids began long ago, perhaps even by E. Rutherford and his co-workers. The passage of nuclear particles through matter was calculated by N. Bohr, H. Bethe, J. Lindhard, O.B. Firsov and others. But the radiation defects (RD) were noticed later, when at first radiation-resistant structural materials for nuclear reactors, and also devices capable of operating under extreme radiation conditions for long exposure times were required. With the miniaturization of semiconductor components, it became clear that nanoscale objects (whose dimensions are comparable to the de Broglie wavelength of conduction electrons) behave under irradiation differently than bulk materials [1-3].

For many years, in the study of radiation effects in solid-state materials, a microscopic approach has been developed which requires detailed information on the nature of RDs, their electronic and thermodynamic parameters, atomic configuration, etc. Just in this direction developed the traditional physics of wide-gap crystals which required a huge amount of experimental measurements and theoretical developments. Meanwhile, a large number of materials, their different history (growing technology, impurity composition), various irradiation conditions (temperature, type of particles and their energy, beam intensity) actually make this approach unrealistic. A change in the subsystem of defects under the influence of radiation (electronic, ionic or neutron) causes a change in the spectrum of localized defect-related states, which leads to a change in the properties of the material. Just this is the reason for the low resistance of optoelectronic devices based on such materials to the exposure of high energy radiation [1-5].

The study of radiation damage to wide-gap corundum single oxide crystals $\left(\alpha-\mathrm{Al}_{2} \mathrm{O}_{3}\right)$ by highenergy electrons, protons, neutrons and heavy particles is of interest related with their practical use in high-radiation areas, for example, in studying the properties of materials under the influence of ionizing effect for nuclear engineering facilities, due to their exceptional properties: high hardness, refractoriness, chemical and radiation resistance, low electrical conductivity and high optical transparency within a wide spectral range. It is also known that one of the consequences of the 
impact of heavy charged particles on solids is the generation of mechanical stresses in the irradiated layer, which, in turn, affect the processes of defect formation [4-8].

When an energetic particle penetrates through matter, it loses energy in two almost independent processes: (i) elastic collisions with nuclei, characterized by nuclear energy losses $(\mathrm{dE} / \mathrm{dx})_{\mathrm{n}}$, that dominate at energies up to $1 \mathrm{keV} / \mathrm{amu}$; (ii) inelastic collisions of a charged particle with electrons of substance atoms known as electron losses $(\mathrm{dE} / \mathrm{dx})_{\mathrm{e}}$, that dominate at energies from $1 \mathrm{MeV} / \mathrm{amu}$ and higher. In inelastic collisions with a typical cross section of $\sim 10^{-16} \mathrm{~cm}^{2}$ energy is transferred from the incident particle to the target atoms through the excitation and ionization of electron shells. The displacement of atoms from the lattice sites occurs due to the transfer of momentum from the incident particle to the atoms of the lattice (nuclear losses). If an atom (or ion) receives enough energy in a collision, it will be displaced from site to interstice, and a vacancy will appear in its place. Such atom can meet another vacancy and recombine with it, or remain in an interstitial position (intrinsic interstice), or be captured by an impurity atom. Vacancies can also be mobile and either create complexes with impurities, or combine into multi-vacancy complexes. Defects that are stable at the irradiation temperature can become mobile with subsequent heating. The mobility of defects is of paramount importance in the evolution of radiation damage in solids.

The total cross section of the displacement of an atom from a lattice site in an elastic collision is given by the following integral

$$
\sigma(E)=\int_{T_{d}}^{T_{m}} d \sigma(E, T)
$$

where $E$ is the kinetic energy of the incident particle, $T$ is the kinetic energy transferred to the target atom, $d \sigma(E, T)$ is the differential cross section of the corresponding interaction. Integration is performed over the energies from the minimum required to displace the atom(ion) to the interstitial position $\left(T_{d}\right)$ to the maximum that can be transferred in a single collision $\left(T_{m}\right) . T_{d}$ is called the threshold displacement energy. The differential cross section depends on the interaction potential. In the case of charged particles (electrons, protons, ions), the interaction can be described by the Coulomb potential; for neutral particles (neutrons), a collision of hard spheres can be considered [512].

It is known that during irradiation of corundum crystals with various types of ionizing ionization (electrons, neutrons or lead ions) the sample is "destroyed" with the formation of anionic vacancies $\mathrm{O}_{\mathrm{i}}{ }^{2}$ and interstitial ions $\mathrm{Al}_{\mathrm{i}}^{3+}$, as well as complex defects. The formation of pre-radiation defects also occurs during crystal growth, when a part of the interstitial ions diffuses to the surface. The breaking of atomic chains on the crystal surface leads, as is known, to the formation of electronic states, as well as to a change in the lattice periodicity and charge effects. The inhomogeneous distribution of defects on the surface, which leads to a change in the reflectivity, confirms that there is a violation of stoichiometry in the surface layers as a result of elastic interaction with the crystalline structure of the material under study and transport of ions to the surface of the material. In this case, some of the oxygen ions are removed from the crystal, since they cannot be accommodated in octahedral voids due to the fact that the ionic radius of oxygen is larger than that of aluminum (1.81 and $0.57 \AA$, respectively). Indeed, the $\mathrm{Al}$ ion resulting from the decomposition of $\mathrm{Al}_{2} \mathrm{O}_{3}$, is apparently located inside the vacancy pores and partially on the crystal surface, filling interstitial loops and creating a "mirror" surface due to the process of radiationstimulated desorption (RSD) [4, 5].

In this work, we investigated the features of the effect of various types of irradiation on radiation-induced processes of destruction of the surface state of corundum single crystals. 


\section{Materials and technique}

We studied corundum single crystals grown in vacuum by horizontal unidirectional crystallization (HUC) and crystals grown by the Czochralski method, in which the concentration of controlled impurities ( $\mathrm{Ti}, \mathrm{Cr}, \mathrm{Mn}, \mathrm{Fe}, \mathrm{Co}$ ) is from $10^{-4}$ to $10^{-3}$ wt.\%. The Czochralski crystals were heat treated in vacuum at a pressure of $1.5 \cdot 10^{3} \mathrm{~Pa}$ at a temperature of $1950{ }^{0} \mathrm{C}$. The samples were irradiated with various types of radiation, for example, $50 \mathrm{MeV}$ electrons "ARUS" linear electron accelerator, $2 \mathrm{MeV}$ reactor neutrons and $3.5 \mathrm{MeV} / \mathrm{amu}$ lead ions. After irradiation with lead ions, samples were heat treated at $2000{ }^{\circ} \mathrm{C}(\mathrm{t}=2$ hours $)$. To avoid heating during irradiation, the samples were placed in a special chamber with liquid nitrogen vapor blowing. Irradiation with oxygen ions was carried out using HVEE heavy ion accelerator. The processes of amorphization of the corundum surface were studied using JEM 2000FX electron microscope (200 keV, $300 \mathrm{~K})$.

The reflection spectra of corundum single crystals were investigated using synchrotron radiation $(\mathrm{SR})$ on a spectral-kinetic measurement installation in the range of $5-30 \mathrm{eV}(\mathrm{E}=$ $680 \mathrm{MeV}$ ) in UV and VUV ranges at various temperatures were carried out using time-resolved SUPERLUMI (HASYLAB, DESY) experimental device.

When identifying the type of defects (vacancy or interstitial ones), the method of defocusing dark-field electron microscopic images was used, which uses differences in the displacements of images of point defects complexes when changing the focal length of the objective lens.

\section{Results and discussion}

It is known that irradiation of $\alpha-\mathrm{Al}_{2} \mathrm{O}_{3}$ with high-energy particles (electrons, neutrons, ions) results in formation of point, complex $\left[\mathrm{Al}_{\mathrm{i}} \mathrm{F}\right]$ and clustered defects. The latter, in particular $\mathrm{Al}_{\mathrm{i}}^{3+}:\left[\mathrm{O}^{2-}\right]_{6}$, are formed in $\mathrm{Al}_{2} \mathrm{O}_{3}$ due to bombardment with neutrons and heavy lead ions. In the irradiated $\mathrm{Al}_{2} \mathrm{O}_{3}$ crystals, the reflection coefficient decreases, which is a confirmation of the effect of irradiation on the crystal surface. Apart from surface defects, the scattering of charge carriers increases, which also contributes to the reflection coefficients of single crystals [4, 15-18].

After electron irradiation, the concentration of point defects is greater than the concentration of radiation clusters, complexes, or interstitial $\mathrm{Al}_{\mathrm{i}}$ and $\mathrm{O}_{\mathrm{i}}$. After neutron irradiation, due to a cascade of elastic collisions, the concentration of knocked-out atoms is higher than under electron irradiation, and will be much higher if we take into account the losses of the main part of the electron energy during inelastic interaction. Therefore, the concentration of defects is not identical.

Besides, there are inelastic interactions which modify the charge state of pre-irradiation defects according to the following reaction: $\mathrm{F}^{2}+\mathrm{e} \rightarrow \mathrm{F}^{+} ; \mathrm{F}^{2}+2 \mathrm{e} \rightarrow \mathrm{F}$, resulting in the formation of $\mathrm{F}-$ and $\mathrm{F}^{+}$- centers as well as other centers.

After irradiation with lead ions (i.e., when an amorphous phase is formed as a result of crystal disordering), there is some probability of atom displacement from lattice site to interstice during heating. This probability is given by $\sim\left(\mathrm{r}^{2} \omega / 2 \pi \mathrm{k}\right) \exp (-\mathrm{HT})$ [19], where $\mathrm{H}$ is the Frenkel pair formation heat, $\omega / 2 \pi \sim 10^{13} \mathrm{~s}^{-1}$ is the vibration frequency of the atom in the lattice.

An assessment of the defect formation in the case of irradiation with lead ions is presented, when the average energy lost by an ion per unit path $d E / d x$ is $42 \mathrm{keV}$ at a dose of $1.2 \cdot 10^{12} \mathrm{ion} / \mathrm{cm}^{2}$. It is shown that during ion exposure, each $\mathrm{Pb}^{208}$ with an energy of $3.5 \mathrm{MeV} / \mathrm{amu}$ initiates a cascade of atomic substitutions: 420 for $\mathrm{Al}$ and 233 for $\mathrm{O}$. It can be seen from these estimates that $\overline{\mathrm{N}} \approx 10^{40}$, therefore the observed decrease in the reflection coefficient at $h v<25 \mathrm{eV}$ is due to the formation of local amorphous zones.

The defect formation process was studied using the method of electron spectroscopy, which made possible to understand the morphology of the surface state of irradiated corundum. Irradiation with $200 \mathrm{keV} \mathrm{O}^{+}$ions allowed to observe this process of structure formation. 
The observed changes in the process of radiation growth can continue until the configuration of the zone becomes energetically unstable, i.e. until it becomes possible to transform it into another defect (dislocation loop or pair). Indeed, the growth of the zone by Al ions and its transformation into a loop occurs due to merging with the newly born zone, which is accompanied by a local release of high energy $[4,5]$.

The average number of point defects formed as a result of irradiation, at which the formation of complex complexes is possible, is calculated using the following formula:

$$
\bar{N}=\frac{2 q}{\omega}=\frac{E_{1}}{E_{d}} \frac{\ln \left(1+\sqrt{\lambda} \frac{E}{E_{1}}\right)}{\sqrt{\frac{E}{\varepsilon_{1}}-\frac{1}{\sqrt{\lambda}}-\frac{E}{E_{1}} \ln \left(1+E_{1} / \sqrt{E \varepsilon_{1}} / 1+E_{1} / E \sqrt{\lambda}\right)}}
$$

Where $\mathrm{q}$ is the number of pairs of point defects created by an ion per unit path, $\omega$ is the number of amorphous zones, $E_{1}$ is the ion energy transferred to the atom, $E_{d}$ is the threshold energy in front of the atom, $\varepsilon_{1}$ is the displacement energy of the atom, $\mathrm{E}$ is the energy of the scattered ion, $\lambda$ is the maximum energy transferred to the atom.

In the process of crystal growth, mechanical disturbances are formed in the form of microcracks, dislocations, vacancy pores, etc. During irradiation with high-energy particles, some filling of such disturbances with aluminum atoms and metallization of the surface itself occurs, and as a result, a change in the reflectance coefficient is observed depending on the radiation dose (Fig. $1)$.

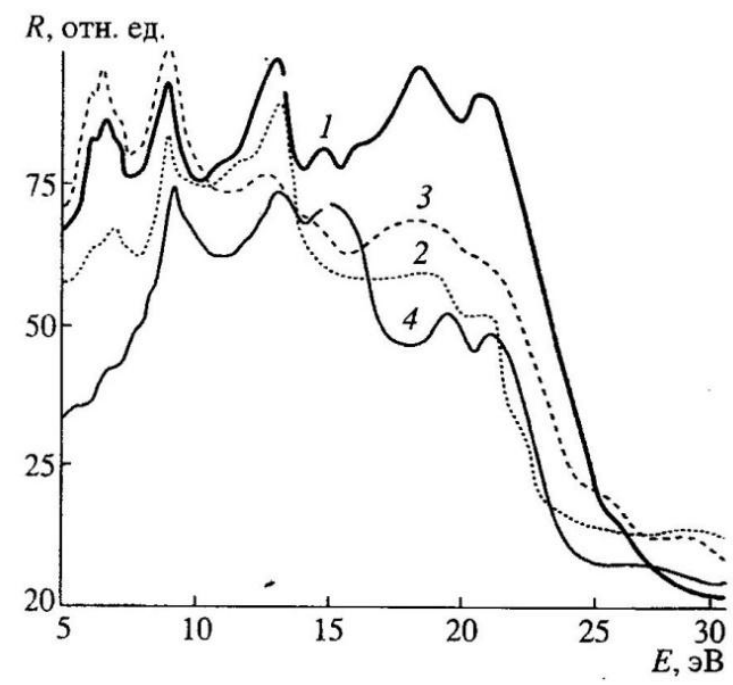

Fig. 1. Reflection spectra of corundum single crystals within the range of 5-30 eV measured at 300K: 1-non-irradiated crystal grown by the horizontal unidirectional crystallization (HUC) method; 2-irradiated at a dose of $1 \times 10^{17} \mathrm{n} / \mathrm{cm}^{2} ; 3$-irradiated at a dose of $1 \times 10^{17} \mathrm{n} / \mathrm{cm}^{2}$ and heat-treated at $500^{\circ} \mathrm{C}$; 4-crystal grown by the Czochralski method and irradiated at a dose of $12 \times 10^{12} \mathrm{~Pb} / \mathrm{cm}^{2}$.

To study the processes of defect formation, the method of electron spectroscopy was used, which made it possible to understand the morphology of the surface state of irradiated corundum, as well as the identification and image of complexes of point defects. In this case, new zones can be generated and disappear or merge yielding the formation of defect complexes [13-18].

This experiment has shown that decrease in $\mathrm{F}^{ \pm}-$center' $(4.1 \mathrm{eV}, 6.05 \mathrm{eV}$ ) band intensity in comparison with that of $\mathrm{F}$ - center takes place due to the formation of free electrons and holes in corundum at irradiation with high-energy SI photons. A part of electrons and holes are captured by single and complex CC (bands in near UV and visual spectral ranges), the others by $\mathrm{F}-$ and $\mathrm{F}^{+}-$ centers. It is found that in the case of neutron irradiation with energy $2 \mathrm{MeV}$ in result of a cascade 
of elastic collisions, the concentration of knocked-on atoms is more than in the case of electron irradiation and should be even more taking into account the losses of the basic part of electron energy at inelastic scattering due to the Coulomb interaction.

Before the collision with the atoms of substances the accelerated electrons lose a considerable part of their energy due to ionization, that is why the number of generated vacancies, oxygen $\left(\mathrm{O}_{\mathrm{i}}\right)$ and aluminum $\left(\mathrm{Al}_{\mathrm{i}}\right)$ interstitial ions in the case of electron irradiation is much less than in the case of neutron irradiation.

The relationship between these processes can be represented by the following expression:

$$
R^{3}=\frac{3}{8} R^{1}\left(r_{0}^{2}+r^{1^{2}}\right)+\frac{1}{2}\left(r_{0}^{3}+r^{1^{3}}\right)+\frac{3}{16} \frac{\left(r^{1^{2}-}-r_{0}^{2}\right)}{r_{0}^{2}}-\frac{R^{1^{3}}}{16}
$$

where $r_{0}$ is the center of the new zone, $r^{1}$ is the existing zone, $r^{1}+r_{0}$ is the disappeared zone, $R$ is the - new zone, $\mathrm{R}^{1}$ is the center of the merge zone.

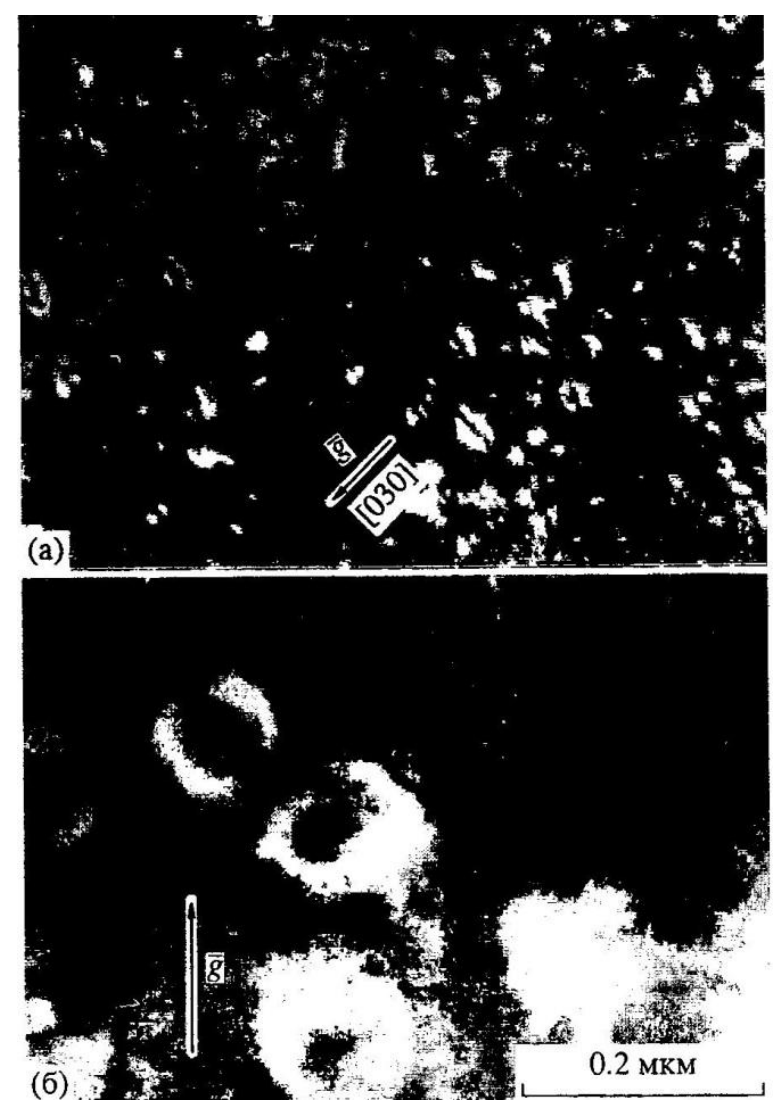

Fig. 2. Defects on the surface of corundum (HUC) irradiated with oxygen ions at a dose of $5 \times 10^{15} \mathrm{ions} / \mathrm{cm}$ a) after 30 min heat treatment at $900{ }^{\circ} \mathrm{C}$; b) after 90 min heat treatment at $900{ }^{\circ} \mathrm{C}$.

It follows from the expression (Fig. 2) that the volume of the radius $\mathrm{R}$ formed as a result of the merging, is equal to the sum of the volumes of the merged zones minus the volume of the overlap area. This assumption is confirmed in the process of defect formation in corundum irradiated with oxygen ions. The indicated process of radiation growth can continue until the configuration of the zone becomes energetically unstable, i.e., until it becomes possible to transform it into another defect, for example, dislocations loop or pore, etc. The growth of the zone by aluminum ions and its transformation into a loop occurs due to merging with the newly generated zone accompanied by a local release of high energy, which creates conditions for the defect region of the crystal to take on a configuration with the lowest energy. 
The radiation growth of the near-surface layer is shown by expression (3) that connects the intensity I of the production of zones of radius $r_{0}$ enriched with free aluminum ions and the number of these zones per unit volume $\mathrm{N}(\mathrm{t})$ :

$$
\frac{d N}{d t}=\operatorname{I\eta }(t)-\frac{4}{3} \pi\left(2 r_{0}\right)^{3} I N(t)
$$

where

$$
\eta(t)=1-\frac{4}{3} \pi\left[\left(2 r_{0}\right)^{3} N(t)+\int_{r_{0}}^{R}\left(r^{1}+r_{0}\right)^{3} n\left(r^{1}, t\right) d r^{1}\right]
$$

Here, $r_{0} N(t) I$ is the coefficient that shows in what part of the volume and in the near-surface layer of the crystal the generation of zones with the initial radius $r_{0}$ can occur.

\section{Conclusions}

Thus, the radiation damage on the crystal surface can have a significant effect on the kinetics of atoms diffusion: because of the presence of the "natural roughness" steps, for example, a nonuniform electric field, etc. is formed. Self-organization of crystals upon irradiation, e.g. by ion beams leads to generation of various nano-periodic structures, for example, arrays. The need to study radiation effects in "quantum-size structures" is primarily due to the emergence of more and more nano-electronic devices and their application in space, nuclear power and elementary particle physics, as well as the widespread use of ion implantation and nuclear doping. One should also not forget about the possibilities of using $\mathrm{RD}$ in instrument technology. Therefore, work in this direction will be enough for many more years and continues to actively develop.

\section{References}

[1] G.W. Arnold, G.B. Kreft, C.B. Norris, Appl. Phys. Lett. 25 (1974) 540.

[2] Yu.S. Kovalev, V.A. Skuratov, S.I. Tyutyunnikov, G. Buinarovskiy, Letters v ECHAYA 7 (2010) 594.

[3] V.V. Harutyunyan, A.K. Babayan, V.A. Gevorkyan, Surface. X-ray, synchrotron and neutron studies 11 (1999) 21.

[4] V.V. Harutyunyan, E.M. Aleksanyan, N.E. Grigoryan, A.A. Sahakyan, G.N. Yeritsyan, Armenian Journal of Physics 10 (2017) 69.

[5] N. Khalfaoui, J.P. Stoquert, F. Haas, C. Traumann, A. Meftah, M. Toulemonde, Nucl. Instr. Meth. B 286 (2012) 247.

[6] R. Chen, Thermally and optically stimulated luminescence: A simulation approach (Chichester: Wiley, 2011).

[7] J.C.R. Mittani, A.A.R. da Silva, F. Vanhavere, M.S. Akselrod, E.G. Yukihara, Nuclear Instruments and Methods in Physics Research Section B: Beam Interactions with Materials and Atoms 260 (2007) 663.

[8] M.S. Kulkarni, M. Luszik-Bhadra, R. Behrens, K.P. Muthe, N.S. Rawat, S.K. Gupta, D.N. Sharma, Nuclear Instruments and Methods in Physics Research Section B 269 (2011) 1465.

[9] N. Itoh, K. Tanimura, Y. Nakai, Nuclear Instruments and Methods in Physics Research Section B: Beam Interactions with Materials and Atoms 65 (1992) 21.

[10] B. Mukherjee, J. Lambert, R. Hentschel, J. Farr, Nuclear Instruments and Methods in Physics Research A 652 (2011) 408.

[11] V.A. Skuratov, A.E. Efimov, D.L. Zagorskiy, Solid State Physics 44 (2002)165.

[12] S.V. Soloviev, I.I. Milman, A.I. Syurdo, Solid State Physics 54 (2013) 683.

[13] V.A. Skuratov, S.J. Zinkle, A.E. Efimov, K. Havancsak, Nucl. Instr. Meth. B 203 (2003) 136.

[14] V.A. Skuratov, S.J. Zinkle, A.E. Efimov, K. Havancsak, Surface and Coating Technology 196 (2005) 56.

[15] V.A. Skuratov, A.E. Efimov, K. Havancsak, Nucl. Instr. Meth. B 250 (2006) 245.

[16] V.A. Skuratov, J. O'Connell, N.S. Kirilkin, J. Neethling, Nucl. Instr. Meth. B 326 (2014) 223.

[17] N. Khalfaoui, J.P. Stoquert, F. Haas, C. Traumann, A. Meftah, M.Toulemonde, Nucl. Instr. Meth. B 286 (2012) 247.

[18] A.A. Leino, S.L. Daraszewicz, O.H. Pakarinen, F. Djurabekova, K. Nordlund, B. Afra, P. Kluth, Nucl. Instr. Meth. B 326 (2014) 289. 